\title{
Correction: Family physician-led, team-based, lifestyle intervention in patients with metabolic syndrome: results of a multicentre feasibility project
}

An earlier version of this article (published Mar. 14, 2017) contained errors in the abstract and results section. ${ }^{1}$

In the abstract results, the sentence "At 12 months, $19 \%$ of patients (95\% confidence interval [CI] 14\%-24\%) showed reversal of MetS, $\mathrm{VO}_{2 \max }$ increased by $16 \%$ (95\% CI 13\%-18\%), and Healthy Eating Index and Mediterranean Diet Scores improved by $9.6 \%$ (95\% CI $7.6 \%-11.6 \%)$ and $1.4 \%(1.1 \%-$ $1.6 \%$ ), respectively." should have read (correction in italics), "At 12 months, $19 \%$ of patients (95\% confidence interval [CI] $14 \%-24 \%$ ) showed reversal of $\mathrm{MetS}, \mathrm{VO}_{2 \max }$ increased by $16 \%$ (95\% CI 13\%-18\%), and Healthy Eating Index and Mediterranean Diet Scores improved by 9.6 (95\% CI 7.6-11.6) points on a 100-point scale and by 1.4 (95\% CI 1.1-1.6) points on a 14-point scale, respectively."

In the main results, "Aerobic capacity and diet quality," the sentence "Both diet quality scores, HEI-C and MDS, improved significantly over time (95\% CI 7.6\%-11.6\% and $1.1 \%-1.6 \%$, respectively) (Table 2)." should have read (correction in italics), "Both diet quality scores, HEI-C and MDS, improved significantly over time (9.6, 95\% CI 7.6-11.6 on a 100-point scale and 1.4, 95\% CI 1.1-1.6, on a 14-point scale, respectively) (Table 2)."

These errors have been corrected at cmajopen.ca.

The authors would also like to clarify that all changes reported in the abstract and body of the manuscript are absolute rather than relative.

\section{Reference}

1. Jeejeebhoy K, Dhaliwal R, Heyland DK, et al. Family physician-led, teambased, lifestyle intervention in patients with metabolic syndrome: results of a multicentre feasibility project. CMAJ Open 2017;5:E229-36.

CMAJ Open 2017. DOI:10.9778/cmajo.20170062 